\title{
LTE Advanced Relaying Standard: A survey
}

\author{
Ghayet el mouna ZHIOUA \\ Telecom ParisTech, \\ Paris, FRANCE \\ zhioua@telecom-paristech.fr
}

\author{
Nabil TABBANE \\ MEDIATRON, Sup'Com, \\ Tunis, TUNISIA \\ nabil.tabbane@ @upcom.rnu.tn
}

\author{
Houda LABIOD \\ Telecom ParisTech, \\ Paris, FRANCE \\ labiod@telecom-paristech.fr
}

\author{
Sami TABBANE \\ MEDIATRON, Sup'Com, \\ Tunis, TUNISIA \\ sami.tabbane@ supcom.rnu.tn
}

\begin{abstract}
LTE Advanced is a well designed system that meets the requirements of $\mathbf{4 G}$ cellular systems. Its standard specification has been lately released. One of its main features is supporting relaying topology inspired from ad hoc multihop networks. This paper is a survey upon the relaying architecture of LTE Advanced standard based on 3GPP Release 10 specifications. A comparison with other relaying architectures, i.e. IEEE $802.16 \mathrm{~m}$ and IEEE $802.11 \mathrm{~s}$ is handled. The originality of this work is in involving comparison with multihop architecture supported by IEEE 802.11s based wireless mesh networks. Several similarities are noted and differences are pointed out.
\end{abstract}

Keywords - LTE Advanced; relaying; multihop architecture; IEEE 802.16m; IEEE802.11s

\section{INTRODUCTION}

International Telecommunication Union - Radio Communication Sector (ITU-R) officially announced future broadband wireless systems as International Mobile Telecommunications-Advanced (IMT-Advanced). It is the $4^{\text {th }}$ generation of cellular systems. IMT-Advanced offers high quality of services and supports enhanced peak data rates in order of $100 \mathrm{Mbit} / \mathrm{s}$ for high-mobility (up to $350 \mathrm{~km} / \mathrm{h}$ ) and 1 $\mathrm{Gbit} / \mathrm{s}$ for low-mobility environments (up to $10 \mathrm{~km} / \mathrm{h}$ ).

In November 2004, Third Generation Partnership Project (3GPP) started the work on the evolution of the 3G cellular systems with a workshop where it defined the Long Term Evolution (LTE) and its Evolved Packet switched System (EPS) architecture. By the end of 2008, LTE and its core network, commonly known as System Architecture Evolution (SAE), specifications have been included in 3GPP Release 8 [1]. SAE offers many advantages over previous and current $3 \mathrm{G}$ systems, e.g. all-IP architecture supporting both IPv4 and IPv6 (see fig. 1), reduced latency, adaptability, scalability, robust radio access technology, reduced deployment and maintenance costs, etc. However, LTE as standardized in 3GPP Release 8 is not a full $4 \mathrm{G}$ standard despite it offers significant performances improvement. Therefore, 3GPP has focused on requirements set by IMT-Advanced project for $4^{\text {th }} \mathrm{G}$ cellular systems and afterward, proposed LTE Advanced as a full $4^{\text {th }} \mathrm{G}$ standard [2]. LTE Advanced, also referred to as 3GPP Release 10, is a well designed and advanced system developed to be backward compatible with existing cellular architecture by just adding some enhancements to LTE Release 8 specifications. It is important from an operator point of view to achieve preexisting compatibility, as it allows soft integration of the new system without facing high new deployment costs and dropping existing investments. LTE Advanced deployment is handled by just software upgrades for most of LTE Release 8 entities. It is also important from user point of view that new proposed systems achieve backward compatibilities with already acquired user's terminal. LTE Release 8 provides only an UL peak rate up to $75 \mathrm{Mbit} / \mathrm{s}$, a DL peak rate up to 300 $\mathrm{Mbit} / \mathrm{s}$ and $4 \mathrm{bit} / \mathrm{s} / \mathrm{Hz}$ for UL peak spectrum efficiency. However, it presents a latency of about $5 \mathrm{~ms}$ and a DL peak spectrum efficiency of $16 \mathrm{bit} / \mathrm{s} / \mathrm{Hz}$ which meets IMT advanced requirements. Besides, LTE Release 8 supports spatial multiplexing and transmission diversity achieved by using MIMO antennas. Beamforming is also handled [6]. With LTE Release 9, achieved at the end of 2009, only few enhancements were added such as support of broadcast and multicast services (E-MBMS), defining new self-optimization features for Self Organizing Network (SON) capabilities, etc. Likely, LTE Release 10 is the evolution of LTE Release 8/9 to meet IMT- 
Advanced requirements. New added features are bandwidth aggregation, enhanced MIMO, cooperative multi-site transmissions, and relaying. In fact, transmission bandwidth is extended up to $100 \mathrm{MHz}$ by aggregating multiple component carriers (CCs), i.e. the basic unit frequency block, for only one joint transmission to/from one UE while using 5 x $20 \mathrm{MHz}-$ CCs. The main advantage is that operators are no more limited by having a single wideband spectrum bandwidth to offer high data rates services, they have the ability to aggregate nonadjacent CCs with different bandwidths. Moreover, LTE Advanced introduces single-user MIMO with up to 4 transmissions to satisfy the $6.75 \mathrm{bit} / \mathrm{s} / \mathrm{Hz}$ of UL peak spectrum efficiency set by IMT-Advanced requirements.

One of the main improvements of LTE Advanced over 3GPP Release 8 is the support of relaying architecture. In fact, 3GPP has started debate and investigation over multihop architecture for its 4G cellular system and, finally, in March 2010, RAN1/RAN2/RAN3 working groups have closed the study by an agreement to support multihop capabilities in the next 3GPP release. Afterward, 3GPP Release 10 supporting relaying architecture has been first standardized in April 2011 [3, 4]. LTE Advanced has now become a commonly accepted standard and relaying becomes one of its main features.

IEEE $802.16 \mathrm{~m}$, also known as Mobile WiMAX has as well introduced relaying abilities in its radio access network for purpose of coverage and capacity enhencements. Mobile WiMAX is based on the IEEE 802.16-2009 standard [5]. It is the first mobile broadband wireless access solution as compared to the previous IEEE 802.16 releases. In fact, first, WiMAX has been approved to become a $3 \mathrm{G}$ standard and belong to the IMT-2000 standards group, in October 2007. Then, IEEE 802.16j working group was created to develop multihop capabilities for WiMAX standards. Afterward, IEEE 802.16j has been standardized as IEEE 802.16-2009. However, for these WiMAX standards, subscribers' mobility is not supported. Therefore, IEEE 802.16 working group has developed an amendment of IEEE 802.16-2009. Thus mobile WiMAX has been proposed to integrate mobility enhancements and to meet the requirements of IMT-Advanced project for $4^{\text {th }} \mathrm{G}$ cellular systems. The working group designs a common radio access technology and a network architecture that enables the convergence of mobile and fixed broadband networks.

Multihop architecture has been similarly discussed in LTE Advanced and IEEE $802.16 \mathrm{~m}$ standards. However, some differences in radio resource control (RRC) and transport layer exist. In this work, we focus on studying multihop LTE network architecture, as specified in LTE Advanced standard $[3,4]$; and we address the multihop architecture design comparison of these two standards. We also handle the comparison with IEEE 802.11s mesh architecture as its multihop architecture has been defined before and can be viewed as the basis of that proposed in both LTE Advanced and IEEE $802.16 \mathrm{~m}$. Therefore, we first give an overview of LTE Advanced relaying architecture features. Then, we compare its multihop architecture to other relaying approaches, i.e. IEEE $802.16 \mathrm{~m}$ and IEEE 802.11 s. Finally, we give conclusions.

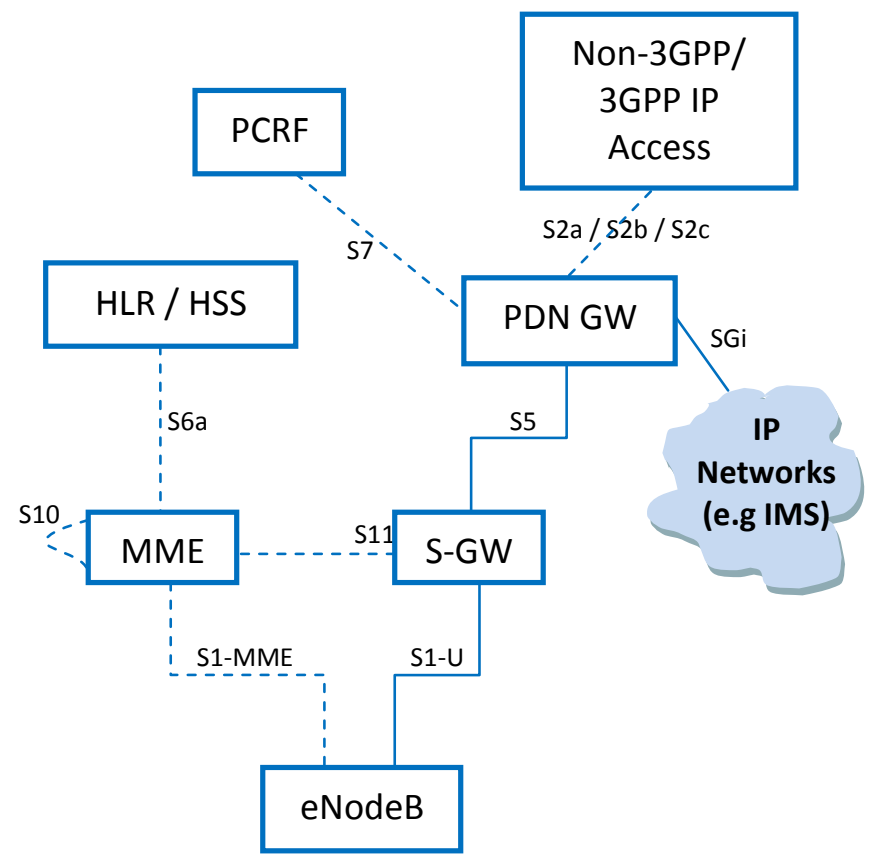

Figure 1. E-UTRAN - EPS Architecture

\section{RELAYING IN LTE ADVANCED}

As coverage problems increase, due to the use of high frequency spectrum and as the capacity improvement could only be achieved by small cell sizes, LTE Advanced has introduced relaying in E-UTRAN. It is also referred to as the multihop architecture because it combines features of both ad hoc and cellular networks. LTE Advanced multihop architecture improves coverage and capacity, especially at cell borders, by reducing transmitting distance through multihop links which achieves higher data rates as compared to the long single links. With relaying, the UE communicates with the network via a relay node that is wirelessly connected to a donor cell using the LTE RAN technology. The donor base station serves one or several relays and mobile station. Reference [24] studies the performance and coverage improvements while introducing relay nodes in an LTE-Advanced network. Amplify-and-forward repeaters and decode-and forward relays strategies are compared. Simulation results show that decode 
and forward relay gives the highest throughput gain and is the best solution for coverage extension. In reference [25], authors investigate in inter-cell interferences in a relay based LTE advanced network. An interference coordination scheme based on prioritized scheduling for relay nodes has been proposed. Simulation results show that the interference coordination improves the system performance in urban scenarios deployments rather than suburban scenarios due to the low received UEs power levels. Reference [26] evaluates the power control scheme proposed in LTE Release 8 standard for a relay based LTE Advanced network. Simulations have shown that the power control increases the cell edge and system capacities and mitigate inter-cell interference. Reference [27] addresses the optimal relay placement issue for purpose of coverage extension by considering both UL and DL transmission scenarios. Reference [28] prove the importance of relay nodes site planning by proposing and analyzing two approaches for relay nodes sites planning. The first approach is location selection and the second is serving cell selection.

The next subsections present standard specifications of LTE Advanced relaying architecture, relay nodes protocols stacks and radio resources partitioning multihop based EUTRAN.

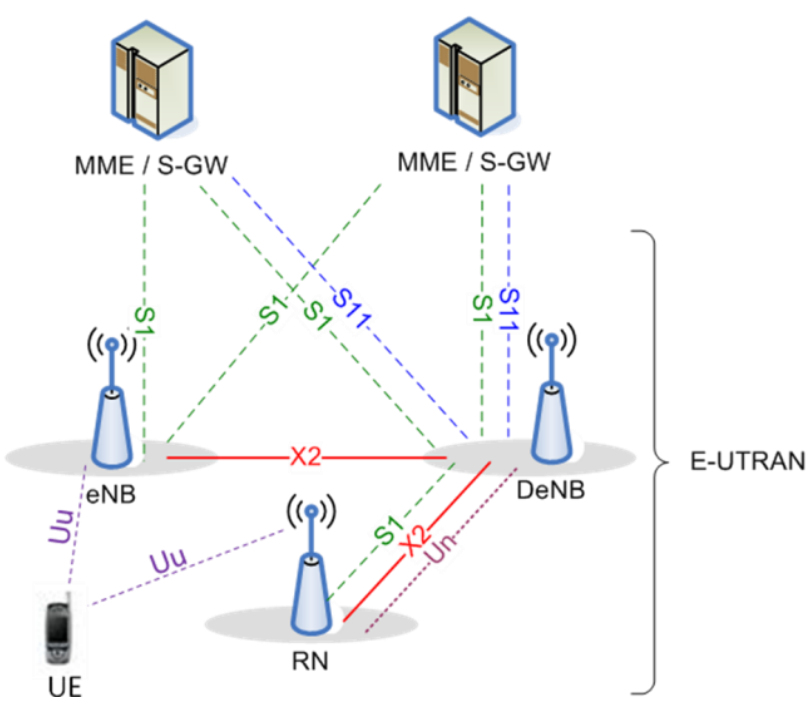

Figure 2. E-UTRAN supporting relaying

\section{A. Architecture}

LTE-Advanced multihop relaying architecture is composed of one relay node (RN) between the eNB and the UE. The RN is wirelessly connected to a donor eNB (DeNB). According to the standard, the access link, i.e. Uu interface, is the link for communication between RN and UE and the backhaul link, i.e. Un interface, is a link for communication between $\mathrm{RN}$ and
DeNB [8]. LTE Advanced RN is a decode-and-forward relay, meaning that the relay node decodes the data from the DeNB, re-encode and then forward it to the UE. A RN includes at least two physical layer entities. One entity is used for communication with its attached UEs, thus it must support eNB functionalities (e.g. Radio Network Layer (RNL) and Transport Network Layer (TNL)). The other physical layer entity, used for wireless communication with its DeNB, handles some UE functionalities (e.g. PHY, RRC, and NAS functionality) extended by relay-specific advancements (e.g. control plane protocols, scheduling capabilities, etc). Moreover, to achieve the backward compatibility, the RN must be able to serve Release 8/9/10 UEs. From a UE perspective, a $\mathrm{RN}$ is considered as a part of the E-UTRAN and behaves like an eNB [3]. It appears to a UE as a distinct cell from the DeNB, and has its own cell Id. Moreover, in addition of supporting radio protocols of the E-UTRA radio interface, the RN supports also protocols of the $S 1$ and $X 2$ interfaces [4]. In fact, the EUTRAN consists of a set of eNBs with fully distributed radio access network architecture. $S 1$ and $X 2$ are logical interfaces [8]. $S 1$ provides an interconnection between the E-UTRAN and the EPC. It interfaces the eNB with the CN. $X 2$ is a logical interface between two eNBs. Although logically representing a point to point link between eNBs, its physical realization need not be necessary a point to point link [8]. According to [9], the separation of $X 2$ Radio Network functionalities and $X 2$ Transport Network functionalities is essential to handle future extension.

Figure 2 illustrates the entities of a multihop base LTEAdvanced E-UTRAN. The neighboring eNBs are interconnected through $X 2$ interface, enabling mutual direct communication. An eNB is connected to the MME/SAE Gateway via the $S 1$ interface. One eNB serves one or more RNs. $S 1$ and $X 2$ functionalities are provided to the RN by its DeNB for inter-connection with other network nodes (e.g. eNBs, MME and S-GWs). Thus DeNB acts like a proxy for the $\mathrm{RN}$. For $S 1$ and $X 2$, the proxy functionalities handled by the DeNB, comprise passing UE-dedicated S1, $X 2$ signaling messages and GTP data packets between $S 1$ and $X 2$ interfaces associated on one side with the RN and on the other side with other network nodes. Due to its proxy functionality, the DeNB appears as an MME (for S1-MME), an eNB (for X2) and an SGW (for S1-U) to the RN. Moreover, the DeNB also handles the S-GW and PDN GW functions needed for the RN operation. It consists of creating a session for the RN, managing RN EPS bearers, terminating the S11 interface towards the MME serving the $\mathrm{RN}$, allocating an IP address for the $\mathrm{RN}$ which may be different from the $S 1$ IP address of the DeNB (it is a native PDN GW function that is performed by the DeNB for its RN). Moreover, RN signaling and data packets are mapped by the 
DeNB onto EPS bearers that are setup for the RN, based on QoS mechanisms defined between UE and PDN GW. Therefore, in addition to the eNB functions, the DeNB hosts S1/X2 proxy functionality and S11 termination and S-GW, PDN GW functionality for supporting RNs [3, 4].

In contrast to ad hoc networks, fixed relay nodes are placed at positions that were planned by the operator in advance and they do not need wired connection to network, thus offering savings in operators' BSs' deployment costs. An eNB can serve one or more multihop links in its cell and a multihop link can comprise two or more hops. However, as the complexity of the multihop cellular network is strongly related to the number of hops, 3GPP has limited the number of hops to two for LTE Advanced [4, 8]. Therefore, the communication between a UE and an eNB can be established either directly through a single hop or over hops via a RN.

In the next subsection, we focus on studying the control plane and user plane protocols of the $X 2$ interface. $X 2$ interface represents a major improvement upon the network architecture of LTE as base stations become directly connected to exchange configuration and management data. With $X 2$ interface, LTE architecture becomes flatter.

\section{B. Self Organizing Network Operations}

Introducing self configuring and self optimizing mechanisms in cellular networks represents an interesting approach to minimize operational effort. In this way, LTE Advanced hybrid ad hoc architecture has been designed to support Self Organizing Network (SON) functionalities. Some SON features are already supported by LTE Release 8 . SON is handled by implementing dynamic optimized processes in the network which increases network reactivity and performances. SON functions can be divided into two categories selfconfiguration and self-optimization. The self-configuration task is a set of functions that enable the network to automatically perform installation procedure, i.e. the plug and play and the self-optimization task is a set of functions that enable the network to auto-tune its operational parameters using UE, eNB and performance measurements. For instance, with a selfconfiguration ability a newly deployed eNB is configured by automatic installation procedures allowing getting the basic configuration necessary for the system to be operational. It is held in the pre-operational state that covers first, the eNB basic setup where the configuration of eNB IP address(es), the association to a S-GW, the downloading of the operational parameters and the eNB software are done. Second, it covers the initial radio configuration parameters where neighbors list configuration and coverage and capacity parameter configuration are set up. Finally, in the self-optimization step, the eNB, based on performance measurements, auto-tune the network. It is the operational state. The eNB does optimization and adaptation functions. The optimization covers the coverage and capacity control and neighbor list optimization. Besides, according to [7], apart from holding self-configuration and optimization functions, LTE Advanced is a SON that provides also the following functionalities: coverage and capacity optimization, inter-cell interference coordination, automatic neighbor relation function, mobility load balancing optimization, energy savings, interference reduction, automated configuration of physical cell Id, mobility robustness optimization, RACH optimization.

\section{RN protocols stack}

The control and user planes protocol stacks of $S 1$ and $X 2$ interfaces are shown in fig.3. The $S 1$ user plane (S1-U) protocol stack for E-UTRAN supporting RNs are shown in (a) S1-U/X2-U protocol stacks. There is a GTP tunnel associated with each UE EPS bearer: from the S-GW associated with the UE to the DeNB, then it is switched to another GTP tunnel in the DeNB, then via one-to-one mapping, it goes from the DeNB to the RN. In the same way, $X 2$ user plane (X2-U) protocol stacks for supporting RNs during inter-eNB handover is similar to the S1-U one, c.f. figure (a) S1-U/X2-U protocol stacks. There is a GTP forwarding tunnel associated with each UE EPS bearer subject to forwarding: from the other eNB to the DeNB, then it is switched to another GTP tunnel in the DeNB, going from the DeNB to the RN via one-to-one mapping. The S1-U and X2-U packets are mapped, based on the QoS Class Identifier (QCI) associated with the UE EPS bearers, to radio bearers. UE EPS bearers with the same QoS are mapped to the same radio bearer. In the same way, $S 1$ and $X 2$ interface signaling packets for control plane are mapped to radio bearers over the Un interface.

The $S 1$ control plane (S1-CP) protocol stacks for supporting RNs are shown in (b) S1 C-plane protocol stacks. There is only one $S 1$ interface relation between the RN and the DeNB, and between the DeNB and each MME in the MME group. The DeNB processes and forwards all S1-AP messages between the $\mathrm{RN}$ and the MMEs for all UE-dedicated procedures. The processing of S1-AP messages means modifying S1-AP UE Ids, Transport Layer address and GTP Ids and leaves other parts of the message unchanged. All S1-AP procedures that are not dedicated to a specific UE are terminated at the DeNB, and handled locally between the RN and the DeNB, and between the DeNB and the MME.

The $X 2$ control plane protocol stacks for supporting RNs are shown in (c) X2 C-plane protocol stacks. There is only one $X 2$ interface relation between the RN and the DeNB, and there 
is one $X 2$ interface relation between the DeNB and every other eNB that the DeNB has an $X 2$ relationship with. The DeNB processes and forwards all $X 2$ messages between the RN and other eNBs for all UE-dedicated procedures. The processing of X2-AP messages means modifying X2-AP UE Ids, Transport Layer address and GTP TEIds and leaves other parts of the message unchanged. All X2-AP procedures that are not dedicated to a specific UE are terminated at the DeNB, and handled locally between the RN and the DeNB, and between the DeNB and other eNBs.

\section{Relay nodes Resource sharing}

Several related works have studied radio resource allocation for relay nodes in an OFDMA based relay-enhanced topology. Withal, for a multihop network, we notice that each resource is partitioned into two transmission phases, where the first phase considers the transmission between the eNB and $\mathrm{RN}$ and the second phase for the RN and UE.

Reference [15] proposes a bandwidth allocation algorithm for relay nodes in a vehicular based multihop topology.

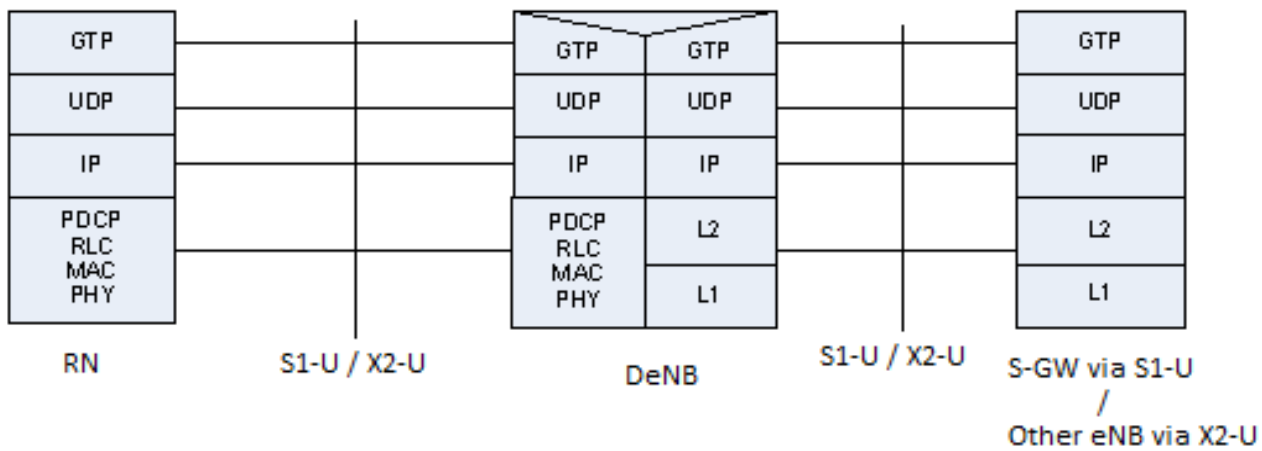

(a) S1-U/X2-U protocol stacks

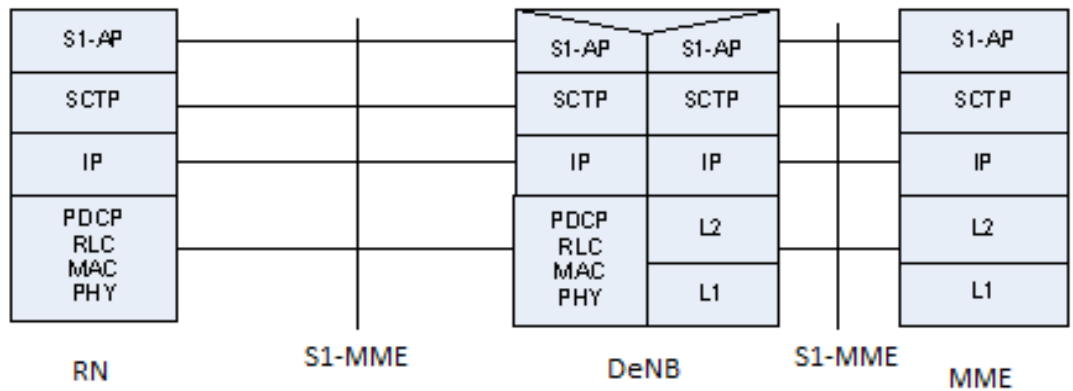

(b) S1 C-plane protocol stacks

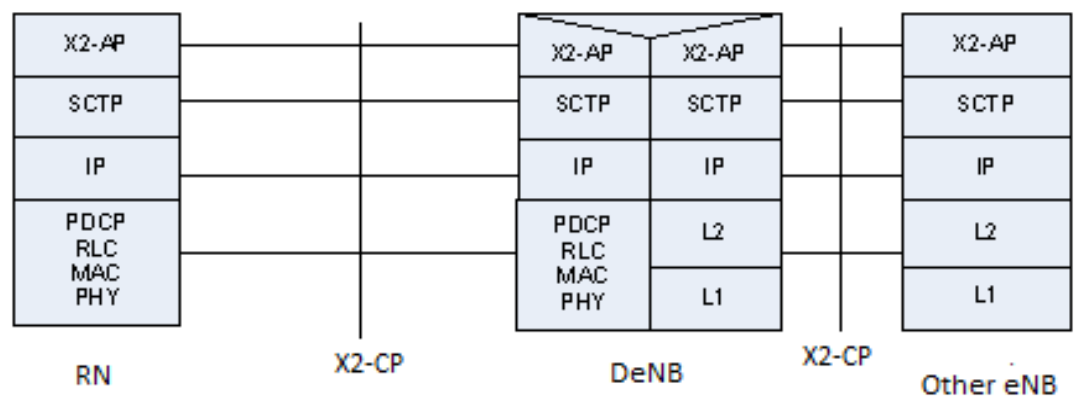

(c) X2 C-plane protocol stacks

Figure 3. Control Plane and User plane protocols stacks for E-UTRAN supporting Relay Nodes 
The algorithm prioritizes traffic flows with the higher priority using service differentiation scheduling. The algorithm proposed in [16] is based on QoS consideration for allocating bandwidth to relays by maximizing a utility function that joins flow's priority and relay node velocity. The utility describes the degree of user satisfaction. Work [17] proposes a distributed method for subchannel allocation based on considering the instantaneous channel conditions and the minimization of a subchannel reuse over the network by forbidding its selection by neighboring cells. Authors in [18] consider multi-service transmission in their relay selection and subcarrier allocation algorithm. A selective relaying mechanism based on capacity maximizing for subcarriers allocation problem is proposed in [19]. Existing research work has also studied power control mechanisms for the downlink transmissions of relay-based OFDMA networks. The works in [20] propose a solution that jointly considers the subchannel and power allocation of a direct transmission and the two-hop path. A dynamic joint subchannel and power allocation scheme is proposed in [21] to maximize the worst user's data rate. Reference [22] proposes a heuristic algorithm to solve the resource allocation problem by satisfying a minimum data rate for every user. A joint relay selection, subcarrier assignment and power allocation problem that achieves proportional fairness is handled in [23].

In this context, LTE advanced standard has defined a framework for integrating relay nodes in the E-UTRAN [3]. In fact, it specifies the OFDMA radio resources used for the access link and backhaul link. Standard denotes that simultaneous DeNB-to-RN and RN-to-UE transmission is not possible because high level of interferences would be experienced between the two physical layer entities composing the node: RN transmitter (for RN-to-UE transmission) and RN receiver (for DeNB-to-RN transmission). Similarly, simultaneous UE-to-RN and RN-to-DeNB is not feasible. This could be overcome by using different bands for access link and backhaul link. However radio resources scarcity and Release 8 backward compatibly make this option impossible. LTE Advanced standard has focused on this challenge. First, it defines that eNB-RN transmissions are in the same band as eNB-UE transmissions. Then it partitions DeNB radio resources and defines a subset of overall resources for backhaul communications. It specifies that eNB-RN transmission must be handled using Multimedia Broadcast multicast service Single Frequency Network (MBSFN) subframes for the purpose of creating a transmission gap in the RN-to-UE transmissions to allow reception of DeNB-to-RN transmissions. DeNB uses then MBSFN subframes during the backhaul communication with the RN and normal subframes to schedule its macroCell users. In an MBSFN subframe, the first one or two OFDM symbols contain Layer 1 and Layer 2 control signaling for access link (e.g. cell-specific reference signals). The rest of the MBSFN subframe can be therefore used for the DeNB-to-RN communication and contains data and DL control information. The standard has defined new control channel for RN signaling, transmitted later in the subframe since control signaling from the DeNB-to-RN carrying the normal control signaling, DL scheduling assignments and UL scheduling grants cannot be transmitted as usual in the first part of an MBSFN subframe. The standard has specified two steps for eNB-RN radio resources selection: subframe selection and symbols selection.

\section{1) Subframes selection}

eNB-to-RN transmissions occur in downlink subframes while RN-to-eNB transmissions occur in uplink subframes, these subframes are configured by higher layers. As specified earlier, DL subframes configured for eNB-to-RN transmission must be configured as MBSFN subframes for the relay node. Subframes that are not MBSFN subframes cannot be used for eNB-RN transmissions. Moreover, radio resources are set through time multiplexing between eNB-RN and RN-UE transmissions. Radio resources allocated to eNB-RN transmissions within the frame depends on its type. In fact, with LTE networks, even the frame structure has been configured to achieve compatibility with existing systems to enable migration from previous systems since both FDD and TDD modes are supported. Frame structure Type 1 is used in FDD mode while Type 2 is deployed with TDD mode. All radio frames, DL and $\mathrm{UL}$ in both modes are of $T_{\mathrm{f}}=307200 \times T_{\mathrm{s}}=10 \mathrm{~ms}$ duration, where $T_{\mathrm{s}}$ is the time unit: $T_{\mathrm{s}}=1 /(15000 \times 2048)$ seconds.

For frame Type 1, UL and DL transmissions are separated in the frequency domain. Therefore, eNB-to-RN and RN-to-UE transmissions occur in the DL frequency band, while RN-toeNB and UE-to-RN transmissions occur in the UL frequency band. Obviously, Type 1 LTE frame is divided into 10 subframes, each subframe is $1 \mathrm{~ms}$ duration. One subframe is further divided into two slots, each of $T_{\text {slot }}=15360 \cdot \mathrm{T}_{\mathrm{s}}=0.5 \mathrm{~ms}$ duration numbered from 0 to 19 [11]. One slot is composed of either 6 or 7 ODFM symbols, depending on whether the normal or extended cyclic prefix (CP) is used (7 symbols for short $\mathrm{CP}$ and 6 symbols for long $\mathrm{CP})$. For eNB-RN radio resources reservation, we always deal with allocating subframes instead of slots. TDD subframes 0 , 1, 5 and 6 and FDD subframes $0,4,5$ and 9 carry system information, synchronization and paging channels, etc. Thus, those subframes cannot be configured as MBSFN subframes because they should be always accessible to the UE. 
For radio frame Type 1, a subframe used for eNB-to-RN transmission must satisfy [12]:

$$
\left[\left(10 \cdot n_{\mathrm{f}}+\left\lfloor n_{\mathrm{s}} / 2\right\rfloor\right) \bmod 8\right] \in \Delta_{\mathrm{BSC}}
$$

The set $\Delta_{\mathrm{BSC}}$ is the offset value for the decision. It is determined as the union of applicable offset values, see Table I, where " $\mathrm{x}$ " means that the corresponding bit can be either 0 or 1. The parameter SubframeConfigurationFDD is configured by higher layers. It defines the DL subframe configuration for eNB-to-RN transmission. In these subframes, DeNB indicates downlink assignments for the RN. Moreover, a subframe $n$ is configured for RN-to-eNB transmission, i.e. UL eNB-RN transmission, if subframe $n-4$ is configured for eNB-to-RN, i.e. DL eNB-RN transmission [12].

The Type 2 LTE radio frame consists of two half-frames of length $153600 \cdot T_{\mathrm{s}}=5 \mathrm{~ms}$ each. Each half-frame is split into five subframes of length $30720 \cdot T_{\mathrm{s}}=1 \mathrm{~ms}$ and is consisted of 10 slots. One slot is $0.5 \mathrm{~ms}$ length and two consecutive slots form one subframe, just like FDD. One of the main advantages of using TDD mode and using Type 2 frame, is dynamically change the UL/DL characteristic configurations to meet traffic load balancing. A total number of seven UL/DL standard configurations (0-6) that use either $5 \mathrm{~ms}$ or $10 \mathrm{~ms}$ switch-point periodicities, have been set within the LTE Advanced standard. To integrate relaying, eNBs resource partitioning for RNs' transmission is performed. Some subframes previously used for eNB-UE communications become dedicated for eNB-RN transmissions. In standard TDD frame, subframes 0 and 5 are used for synchronization, the transmission of $\mathrm{PSCH}, \mathrm{SSCH}$ and broadcast channel $\mathrm{PBCH}$. Subframes 1 and 6 are used for paging. Thus, these subframes 0,1,5,6 cannot be configured as MBSFN subframes as they carry important system information. Table II lists subframes allocated for Un interface; where " $\mathrm{D}$ " means the subframe is configured for DL transmissions (eNB-to-RN) and " $U$ " means the subframe is configured for UL transmissions (RN-to-eNB). The choice of which UL/DL configuration to use is handled by higher layers. While there are 7 possible UL/DL subframes configuration, standard has specified that only subframe configuration 1,2,3,4 and 6 could be used as backhaul transport in the relaying architecture. In fact, with TDD UL/DL configuration \#0, \{DL, UL\} subframes' sets, achieved through time relation computation between UL grant, DL Data transmission and UL Data transmission, UL ACK/NACK, contain these subframes pairs: $\{0,4\},\{1,7\},\{5,9\},\{6,2\}$. However, after excluding subframes $0,1,5,6$ no other subframes can be configured as MBSFN subframe to use for backhaul transmission.
Consequently, UL/DL configuration \#0 could not support relaying. With UL/DL configuration \#5, there is only one subframe for UL transmission (subframe $\mathrm{n}^{\circ} 2$ ), while excluding $\mathrm{S}$ subframes. Therefore, there is no extra subframes that could be used for Un transmissions. A total of 19 eNB-RN UL/DL configurations are possible where a least one UL and DL subframe and up to two UL subframes and three DL subframes are allocated to eNB-RN transmission. For instance, with UL/DL configuration \#1, while ignoring $S$ subframes, DL subframes 4 and 9, respectively UL subframes 3 and 8 could be allocated to the backhaul link. Consequently, for a symmetric allocation, the DL/UL subframe pairs are $\{4,8\},\{9,3\}$ or $\{(4$, $9),(8,3)\}$. For an asymmetric allocation, $\{(4,9), 3\}$ or $\{(4,9)$, $8\}$ are subframes defined for Un transmission.

\section{2) Symbols selection}

After selecting subframe for eNB-RN transmissions, inside each dedicated subframe, eNB-RN transmissions are restricted to a subset of the OFDM symbols in each of the two slots composing the subframe. The starting and ending OFDM symbols respectively in the first and second slot of a subframe is given in Table III. The choice of which configuration to use is handled by higher layers. The simultaneous use of configuration 0 in Table III is not supported by the standard.

\section{LTE RELAYING VS OTHER RELAYING APPROACHES}

As it was described in earlier sections, multihop architecture is one of the key specifications of LTE Advanced standard. In the same way, IEEE $802.16 \mathrm{~m}$ standard has integrated multihop capabilities in its architecture. In this section, an overview of IEEE $802.16 \mathrm{~m}$ relaying specifications and IEEE $802.11 \mathrm{~s}$ architecture is given. Then a comparison between these latter and 3GPP LTE Advanced standard is handled.

\section{A. IEEE $802.16 m$ relaying specifications}

With mobile WiMax, a relay node is referred to as Advanced Relay Station (ARS) and the serving base station is called Advanced Base Station (ABS). The mobile user, referred to as Advanced Mobile Station (AMS), can be attached either to an ABS or to an ARS, see fig. 4. Two relay's categories are defined depending on whether the relay node can generate cell control signaling and manage the resources or not. A nontransparent (NT) relay is an ARS having scheduling capabilities for its attached AMS. Moreover, it has its own PHY Cell Id and independently generates its cell control messages. It is equivalent to the RN Type 1 in 3GPP LTE specifications. A transparent (T) relay (Type 2 relay for 3GPP) is managed and dependent to its serving base station, by sharing the same PHY Cell Id and control messages [13]. 


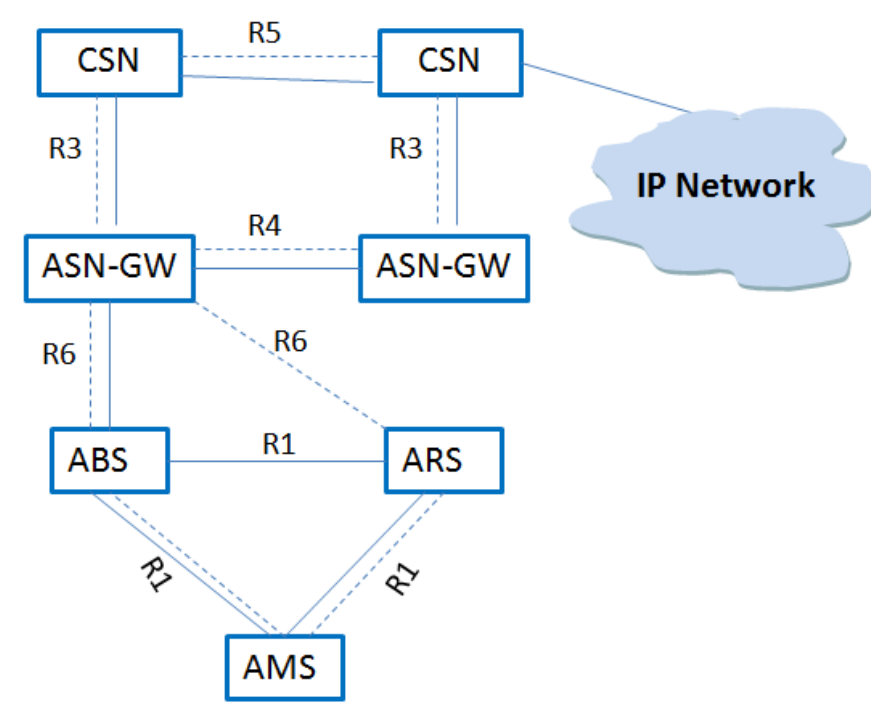

Figure 4. $802.16 \mathrm{~m}$ architecture

For mobile WiMAX, an ARS is a fixed NT station that holds its radio resources scheduling function. It may manage one or more sectors with a unique PHY Cell Id for each controlled sector. Only two hops are allowed with ARSs. The ARS controls its cells and has a wireless backhaul connection to the core network (i.e. ASN-GW) through the ABS [13]. The ARS is transparent from an AMS perspective, i.e. it is considered as an ordinary base station. ASN-GW controls also the ARS like an ordinary ABS and same R1 and R6 mechanisms are used for both.

With $802.16 \mathrm{~m}$, the same radio frame structure supports the both duplex modes TDD and FDD. The frame is of $5 \mathrm{~ms}$ duration, (4 frames are grouped to form a superframe of $20 \mathrm{~ms}$ duration). One frame is further divided into 8 subframes which are assigned to DL or UL transmissions. To integrate relaying operation into the RAN, radio frame is divided into access zone and relay zone. In the access zone, the ABS and ARS transmit to, or receive from, the AMSs. In the relay zone, the ABS transmits to, or receives from, the ARSs and AMSs the ARSs and AMSs. Besides, the access zone part always precedes the relay zone part. It is the serving ABS that informs its ARSs and AMSs of the configuration of the frame structure for relay and access zones. The relay zone contains also the advanced MAP (AMAP) control channel and the data channel, like in the access zone. With $802.16 \mathrm{~m}$ relaying based architecture, several functions are provided such as power control, HARQ, link adaptation, security management. Moreover, it offers location based and enhanced multicast broadcast services. It also supports also femto ABS, multi user and single user MIMO, etc [5].

\section{B. IEEE 802.11s Specifications}

Three types of ad hoc networks could be distinguished according to their application: wireless sensor networks (WSN), mobile ad hoc networks (MANET / VANET) and wireless mesh networks (WMN). The key characteristic of ad hoc networks is their autonomy of being built without relying of any preexisting infrastructure. Minimal configuration and quick deployment make them suitable for many situations especially in emergency cases like natural disasters. All these features make ad hoc networks attractive for an operator to deploy relay stations between the base station and the mobile station in a multihop manner.

The IEEE 802.11s mesh network specification is not finalized yet. It is an amendment to the IEEE 802.11 WLAN standard. It integrates mesh networking services at the MAC layer and is transparent to higher layers protocols.

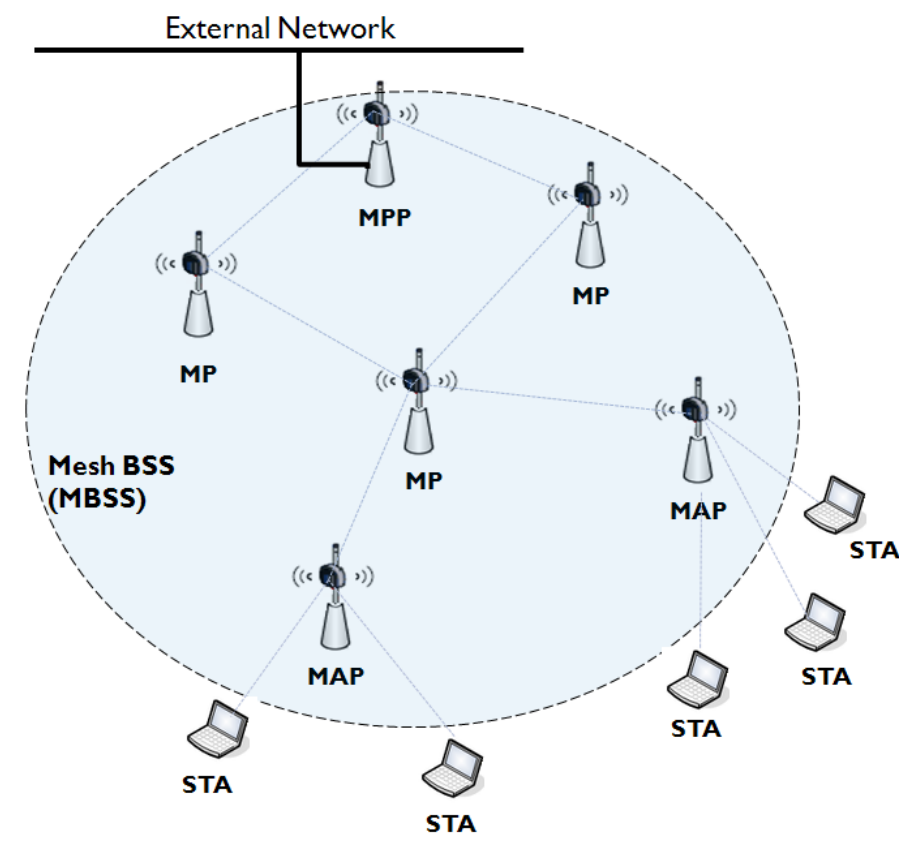

Figure 5. IEEE 802.11s network architecture

The principal entity is the Mesh Point (MP). It may be collocated with one or more other entities (e.g. Access Points (APs), Gateways). An example of $802.11 \mathrm{~s}$ wireless mesh network is illustrated in fig. 5. The Mesh Basic Service Set (MBSS) is composed of peering mesh nodes that are interconnected and communicate through multihop links. It can be composed of Mesh Points (MP), Mesh Portals (MPP) and Mesh Access Points (MAP) to which Stations (STA) could be attached to. The MPP connects the MBSS to external networks.

The MAP provides simultaneously both mesh and AP functionalities. An MBSS is an autonomous wireless mesh network that can be formed without the need of any 
infrastructure. As nodes communicate through multihop links, a routing protocol is needed. MBSS functionalities include: coordination, power management, discovery, security, beaconing and synchronization, channel switching, path selection and forwarding, interworking with external networks and intra-mesh congestion control [14].

\section{Comparison}

This section points out the main differences between relaying in LTE Advanced, 802.16m and 802.11s standards in term of architecture design, system main features, and remaining issues.

\section{1) Architecture Design}

Multihop cellular network architecture enables the operator to increase the system capacity by creating hotspot solutions with reduced interference. For instance, the deployment of relay nodes is simple and could be used even for a temporary traffic load requirement such as non-common events, concerts, etc. Moreover, as relay nodes transmit power is highly lower than macro-BS's, each relay node would cover small areas allowing better channel quality which increases the amount of users under good channel conditions. On the other hand, when base stations' deployment is not possible, a coverage extension for isolated area using relay nodes represents a good solution. Thus, with multihop relays the macro cell coverage can be expanded to the places where the base station cannot reach.

The LTE Advanced RN specifications were handled in the second section. For mobile WiMAX, an ARS is a fixed NT relay node that has scheduling capabilities for its AMSs. It may manage one or more sectors with a unique PHY Cell Id for each sector. ARS has a wireless backhaul connection to the ASN-GW through the ABS. Like in LTE Advanced, from AMS perspective, ARS is considered as an ordinary base station. ASN-GW controls also ARS like an ordinary ABS and same R1 and R6 mechanisms are used for both. With 802.11s, a MBSS is composed of peering MPs communicating through multihop links.

Both $802.16 \mathrm{~m}$ and LTE Advanced allow only two hops communications and don't support RNs' mobility. 802.11s also doesn't support mobile relay nodes but multihop ability isn't constrained by any number of hops. With LTE Advanced, X2 and $S 1$ control interfaces are based on SCTP. X2 control plane protocol stacks are defined between eNB-eNBs and RN-eNB. Data link layer can support any DLL protocol, e.g. PPP, Ethernet, etc. In IP layer, eNB supports IPv6 and/or IPv4. Transport network layer is built on SCTP on top of IP and supports X2 signalling messages exchange between two eNBs. According to [10], there shall be only one SCTP association established between one eNB pair. Moreover, during this SCTP association establishment, if the initiating eNB has more than one IP address, i.e. a multi-homed eNB, the latter must provide to the correspondent eNB the set of its IP addresses. The application layer signalling protocol is referred to as $X 2$ Application Protocol (X2-AP). The X2-AP protocol handles intra LTE mobility management (e.g. handover preparation, context transfer and control of X2-U tunnels from source eNB to target eNB, handover cancellation), load management, reporting errors situations and other $X 2$ general management functions (e.g. setting and resetting the $X 2$ interface, updating the eNB configuration, Inter-cell Interference Coordination). These X2-AP functions are handled via Elementary Procedures (EPs).With $802.16 \mathrm{~m}, R 6$ over $R l$ is based on UDP. For user plane both are based on UDP. With LTE Advanced, The transport for data streams on the $X 2$ interface is based on GPRS Tunnelling Protocol (GTP-U) over UDP over IP. As the transport network layer is built on IP transport over the $X 2$ interface, at the IP layer eNBs supports fragmentation and assembly of GTP packets. An eNB may have one or several IP addresses, that could be IPv6 and/or IPv4 addresses. GTP-U is based on UDP which provides non guaranteed delivery of user plane PDUs.

Relaying capabilities are handled using inband resources for mobile WiMAX and LTE Advanced with adding some specifications like using MBSFN subframes for the latter. With mobile WiMAX, radio frame is divided into access zone and relay zone where the access part always precedes relay zone part. ARS freely uses resources which could be same or different than AMS ones. In the access zone, ABS and ARS transmit to/or receive from, AMSs. In the relay zone, the ABS transmits to/or receives from, ARSs and AMSs The relay zone contains also the advanced MAP (AMAP) control channel and the data channel, like in the access zone. Serving ABS informs its ARSs and AMSs of the configuration of the frame structure for relay and access zones.

With $802.16 \mathrm{~m}$ relaying based architecture, several functions are provided such as power control, HARQ, link adaptation, security management. Moreover, it offers location based and E-MBMS and supports femto ABS, multi user and single user MIMO, etc [5]. MBSS functionalities include: coordination, power management, discovery, security, beaconing and synchronization, channel switching, path selection and forwarding, interworking with external networks and intra-mesh congestion control [10]. Further architecture design information for LTE Advanced, mobile WiMAX and 802.11s networks are presented in Table IV. 


\section{2) System Features}

Table $\mathrm{V}$ presents a detailed comparison in system main features between relaying architecture in LTE Advanced, mobile WiMax and Mesh networks. The comparison includes resources management and scheduling, signaling, handover procedure, power management, and security. With $802.16 \mathrm{~m}$, radio resource configuration is done by the ABS for ARSs communications and relay zone configuration is not frozen. However, with LTE Advanced, radio resources for RNs communications are restricted to be first within MBSFN subframes and then to be within the subset of radio resources selected by the standard for eNB-RN communications. With 802.11s, multiple access with multiple channels is used. Radio resources scheduling is performed by $802.16 \mathrm{~m}$ relay stations. However, LTE Advanced standard has not specified if it is done by RN or ordered by DeNB. Signaling procedures are quite similar for the three. Power management, QoS, synchronization and security mechanisms are described in details in Table V.

\section{3) Open issues}

Some concerns for relaying in LTE Advanced need further studies. Interworking and handovers between $2 \mathrm{G} / 3 \mathrm{G}$ and $4 \mathrm{G}$ systems is not yet optimized. Moreover, 3GPP Release10 standard has defined radio resources partitioning for $\mathrm{RN}$ among DeNB resources'. However, one eNB can serve one or more multihop links. Therefore, scheduling capabilities among RNs are required. Besides, LTE Advanced standard has introduced relaying capabilities without considering RNs' mobility. In fact, in contrast with ad hoc networks, fixed RNs are placed at positions that were planned by the operator in advance. Consequently, planning process remains an operational effort for the operator. Thus, RN autonomy and mobility could introduce dynamicity in LTE multihop architecture. Moreover, decision coordination of when a UE must be attached to the nearest RN must be handled by DeNB. Decision criteria should be selected. From a UE perspective, attachment procedure to RNs must be transparent and efficient.

Many other issues for integrating relaying in LTE Advanced remains; for instance, power saving, relaying cooperative transmission, interworking between relayed and not relayed architectures (e.g. E-UTRAN and mobile WiMAX radio technologies), etc. These issues are not yet addressed.

\section{CONCLUSION}

3GPP Release 10 specifications have been lately released. An overview of the LTE Advanced standard is presented in this paper. Improvements of this release over previous releases are listed. One of its key characteristics is the support of relaying architecture to circumvent the coverage and capacity requirements. Therefore, a survey over LTE Advanced relaying architecture is given. The protocol stacks and RRC for RNs have been defined. In this work, we focused on inbound relays where resources are partitioned between eNodeBs and relay nodes as specified in 3GPP Release 10 standard. There is also outbound relay which is another important way to allocate resources to relay nodes. We provide a comparison between LTE Advanced, IEEE 802.16m and IEEE 802.11s in terms of the proposed relaying architecture. We highlighted similarities and differences focusing on architecture entities and main procedures. We also pointed out remaining issues facing relaying capabilities for LTE Advanced.

\section{REFERENCES}

[1] www.3gpp.org.

[2] ITU-R M.2134, "Requirements Related to Technical Performance for IMT-Advanced Radio Interface(s)"; available for purchase at: http://www.itu.int/dms pub/itu-r/opb/rep/R-REP-M.2134-2008-PDFE.pdf

[3] The $3^{\text {rd }}$ Generation Partnership Project TS 36.216 V10.3.0. June 2011

[4] The $3^{\text {rd }}$ Generation Partnership Project TS 36.300 V10.4.0. June 2011

[5] IEEE $802.16 \mathrm{~m}$ System Description Document, IEEE $802.16 \mathrm{~m}$ 09/0034r4 , 21 Dec 2010; available for purchase at: http://www.ieee802.org/16/tgm/index.html

[6] The $3^{\text {rd }}$ Generation Partnership Project TS 36.423 V10.2.0. Dec 2011

[7] The $3^{\text {rd }}$ Generation Partnership Project TR 36.902 V9.3.1. March 2011

[8] The $3^{\text {rd }}$ Generation Partnership Project TS 36.401 V10.4.0. June 2012

[9] The $3^{\text {rd }}$ Generation Partnership Project TS 36.420 V10.2.0 Sep 2011

[10] The $3^{\text {rd }}$ Generation Partnership Project TS 36.422 V10.1.0. June 2011

[11] The $3^{\text {rd }}$ Generation Partnership Project TS 36.211 V10.2.0. June 2011

[12] The $3^{\text {rd }}$ Generation Partnership Project TS 36.116 V0.2.0. Marsh 2011

[13] Loa, K.; Chih-Chiang Wu; Shiann-Tsong Sheu; Yifei Yuan; Chion, M.; Huo, D.; Ling Xu; "IMT-Advanced Relay Standards", IEEE Communications Magazine, Vol.48, Issue 8, 2010, pp. $40-48$

[14] IEEE P802.11s/D11.0,Draft Standard for Information TechnologyTelecommunications and information exchange between systems-Local and metropolitan area networks - Specific requirements, Part 11: Wireless LAN Medium Access Control (MAC) and Physical Layer (PHY) specifications, Amendment 10: Mesh Networking, April 2011.

[15] Fei, Ridong ; Yang, Kun ; Ou, Shumao; "A QoS-Aware Dynamic Bandwidth Allocation Algorithm for Relay Stations in IEEE 802.16jBased Vehicular Networks", Wireless Communications and Networking Conference WCNC 2010 IEEE, 18-21 April 2010, pp. 1-6

[16] Fei, Ridong ; Yang, Kun ; Ou, Shumao; Xueqi Cheng ; "A QoS-aware Dynamic Bandwidth Allocation Algorithm for Base Stations in IEEE 802.16j-based Vehicular Networks", GLOBECOM 2010, 6-10 Dec. 2010, pp. 1-6

[17] Fraimis I.G. ; Papoutsis, V.D. ; Kotsopoulos, S.A. ; "A Decentralized Subchannel Allocation Scheme with Inter-Cell Interference Coordination (ICIC) for Multi-Cell OFDMA Systems", GLOBECOM 2010, 2010 IEEE Global Telecommunications Conference, 2010, pp.1-5

[18] Z. Danhua, W. Youzheng, and L. Jianhua, "QoS aware relay selection and subcarrier allocation in cooperative OFDMA systems," IEEE Commun.Lett., vol. 14, 2010, pp. 294-296

[19] Hasan, Z. ; Hossain, E. ; Bhargava, V.K. ; "Resource Allocation for Multiuser OFDMA-Based Amplify-and-Forward Relay Networks with Selective Relaying", Communications (ICC), 2011 IEEE International Conference, 5-9 june 2011, pp. 1-6

[20] N. U. Hassan and M. Assaad, "Resource Allocation in Multiuser OFDMA System: Feasibility and Optimization Study," in Proc. IEEE WCNC, Apr. 2009, pp.1-6 
[21] L. Hongxing, L. Hanwen, W. Xinbing, L. Chengyu, and L. Chisheng, "Fairness-Aware Resource Allocation in OFDMA Cooperative Relaying Network," in Proc. IEEE ICC, 2009, pp. 1-5.

[22] S. Woochul, H. Younggoo, and K. Sehun, "Fairness-Aware Resource Allocation in a Cooperative OFDMA Uplink System," IEEE Trans. Veh.Technol., vol. 59, 2010, pp. 932-939.

[23] W. Liping, J. Yusheng, and L. Fuqiang, "Joint Optimization for Proportional Fairness in OFDMA Relay-Enhanced Cellular Networks," in Proc. IEEE WCNC, 2010, pp. 1-6.

[24] Letian Rong; Elayoubi, S.E.; Haddada, O.B. , "Impact of relays on LTEAdvanced performance", ICC 2010, pp. 1-6

[25] Zhe Ren; Saleh, A.B.; Bulakci, O.; Redana, S.; Raaf, B.; Hamalainen, J. , "Joint Interference Coordination and Relay Cell Expansion in LTEAdvanced Networks", IEEE WCNC 2012, pp. 2874 - 2878
[26] Bulakci, O.; Redana, S.; Raaf, B.; Hamalainen, J. , "Impact of Power Control Optimization on the System Performance of Relay Based LTEAdvanced Heterogeneous Networks", Jounrnal of Communications and Networks, Vol.13, Issue.4, Aug 2011, pp. 345-359

[27] Khakurel, S.; Mehta, M.; Karandikar, A., "Optimal Relay Placement for Coverage Extension in LTE-A Cellular Systems", NCC 2012, pp. 1-5

[28] Bou Saleh, A.; Bulakci, O.; Hämäläinen, J.; Redana, S.; Raaf, B. "Analysis of the Impact of Site Planning on the Performance of Relay Deployments", IEEE Transaction on Vehicular Technology, Vol. 61, Issue. 7, Sep 2012, pp. 3139 - 3150

\begin{tabular}{|c|c|}
\hline SubframeConfigurationFDD & $\begin{array}{c}\text { Offset value } \\
\text { element of } \Delta_{\mathrm{BSC}}\end{array}$ \\
\hline$\{x x x x x x x 1\}$ & 7 \\
\hline$\{x x x x x x 1 x\}$ & 6 \\
\hline$\{x x x x x 1 x x\}$ & 5 \\
\hline$\{x x x x 1 x x x\}$ & 4 \\
\hline$\{x x x 1 x x x x\}$ & 3 \\
\hline$\{x x 1 x x x x x\}$ & 2 \\
\hline$\{x 1 x x x x x x\}$ & 1 \\
\hline$\{1 \times x x x x x x\}$ & 0 \\
\hline
\end{tabular}

TABLE I. DL SUBFRAME CONFIGURATION FOR ENB-TO-RN TRANSMISSION FOR TYPE1 FRAME [3] 


\begin{tabular}{|c|c|c|c|c|c|c|c|c|c|c|c|}
\hline \multirow[t]{2}{*}{ SubframeConfigurationTDD } & \multirow{2}{*}{$\begin{array}{c}\text { eNB-RN UL/DL } \\
\text { configuration }\end{array}$} & \multicolumn{10}{|c|}{ Subframe number $n$} \\
\hline & & 0 & 1 & 2 & 3 & 4 & 5 & 6 & 7 & 8 & 9 \\
\hline 0 & \multirow[t]{5}{*}{1} & & & & & $\mathrm{D}$ & & & & $\mathrm{U}$ & \\
\hline 1 & & & & & $U$ & & & & & & $\mathrm{D}$ \\
\hline 2 & & & & & & $\mathrm{D}$ & & & & $\mathrm{U}$ & $\mathrm{D}$ \\
\hline 3 & & & & & $\mathrm{U}$ & $\mathrm{D}$ & & & & & $\mathrm{D}$ \\
\hline 4 & & & & & $\mathrm{U}$ & $\mathrm{D}$ & & & & $\mathrm{U}$ & $\mathrm{D}$ \\
\hline 5 & \multirow[t]{6}{*}{2} & & & $\mathrm{U}$ & & & & & & $\mathrm{D}$ & \\
\hline 6 & & & & & $\mathrm{D}$ & & & & $\mathrm{U}$ & & \\
\hline 7 & & & & $\mathrm{U}$ & & $\mathrm{D}$ & & & & $\mathrm{D}$ & \\
\hline 8 & & & & & $\mathrm{D}$ & & & & $\mathrm{U}$ & & $\mathrm{D}$ \\
\hline 9 & & & & $\mathrm{U}$ & $\mathrm{D}$ & $\mathrm{D}$ & & & & $\mathrm{D}$ & \\
\hline 10 & & & & & $\mathrm{D}$ & & & & $\mathrm{U}$ & $\mathrm{D}$ & $\mathrm{D}$ \\
\hline 11 & \multirow[t]{2}{*}{3} & & & & $\mathrm{U}$ & & & & $\mathrm{D}$ & & $\mathrm{D}$ \\
\hline 12 & & & & & $U$ & & & & $D$ & $D$ & $D$ \\
\hline 13 & \multirow[t]{5}{*}{4} & & & & $U$ & & & & & & $\mathrm{D}$ \\
\hline 14 & & & & & $U$ & & & & $\mathrm{D}$ & & $\mathrm{D}$ \\
\hline 15 & & & & & $\mathrm{U}$ & & & & & $\mathrm{D}$ & $\mathrm{D}$ \\
\hline 16 & & & & & $\mathrm{U}$ & & & & $\mathrm{D}$ & $\mathrm{D}$ & $\mathrm{D}$ \\
\hline 17 & & & & & $U$ & $D$ & & & $D$ & $\mathrm{D}$ & $\mathrm{D}$ \\
\hline 18 & 6 & & & & & $U$ & & & & & $\mathrm{D}$ \\
\hline
\end{tabular}

TABLE II. SUbFAME CONFIGURATION FOR TYPE2 Frame [3]

\begin{tabular}{|c|c|c|c|}
\cline { 2 - 4 } \multicolumn{1}{c|}{} & Configuration & $\begin{array}{c}\text { DL- } \\
\text { StartSymbol }\end{array}$ & $\begin{array}{c}\text { End symbol } \\
\text { index }\end{array}$ \\
\hline \multirow{3}{*}{ First Slot } & 0 & 1 & 6 \\
\cline { 2 - 4 } & 1 & 2 & 6 \\
\cline { 2 - 4 } & 2 & 3 & 6 \\
\hline \multirow{2}{*}{$\begin{array}{c}\text { Second } \\
\text { Slot }\end{array}$} & 0 & 0 & 6 \\
\cline { 2 - 4 } & 1 & 0 & 5 \\
\hline
\end{tabular}

TABLE III. $\quad$ OFDM SYMBOLS IN THE FIRST AND SECOND SLOTS [3] 


\begin{tabular}{|c|c|c|c|}
\hline & IEEE 802.16m & LTE Advanced & IEEE 802.11s \\
\hline Base Station Name & $\mathrm{ABS}$ & DeNB & MP, MAP \\
\hline $\begin{array}{l}\text { Subscriber station } \\
\text { name }\end{array}$ & AMS & UE & Mesh STA or STA \\
\hline $\begin{array}{l}\text { Relay / Backhaul link } \\
\text { interface }\end{array}$ & $\mathrm{R} 1$ & Un & 802.11 s or $802 . \mathrm{X}$ \\
\hline Access link interface & R1 & $\mathrm{Uu}$ & 802.11 PHY/MAC \\
\hline Relays' Mobility & Fixed & Fixed & Fixed \\
\hline Hops' Number & Two hops & Two hops & Unlimited \\
\hline Control plane & R6 over R1 based on UDP & $\mathrm{X} 2$ and $\mathrm{S} 1$ based on SCTP & Not specified \\
\hline User plane & UDP & UDP & Not specified \\
\hline Frame structure & One Type for FDD / TDD & $\begin{array}{l}\text { Type } 1 \text { for FDD mode } \\
\text { Type } 2 \text { for TDD mode }\end{array}$ & 802.11s MAC frame \\
\hline $\begin{array}{l}\text { Radio Resources } \\
\text { partitioning }\end{array}$ & $\begin{array}{l}\text { - Sharing: } \\
\text { ABS, ARS radio resources } \\
\text { are in the same band } \\
\text { - Frame divided into: } \\
\text { * Access Zone } \\
\text { * Relay Zone } \\
\text { - Transmission/Reception: } \\
\text { * Access Zone: } \\
\text { ABS/ ARS to/from AMS } \\
\text { * Relay Zone: } \\
\text { ABS to/from AMS/ARS }\end{array}$ & $\begin{array}{l}\text { - Sharing: } \\
\text { DeBS, RN radio resources are in } \\
\text { the same band } \\
\text { - Relaying over MBSFN } \\
\text { subframes } \\
\text { - Transmission/Reception through } \\
\text { time multiplexing between: } \\
\text { * DeNB-to-RN and RN-to-UE } \\
\text { * UE-to-RN and RN-to-DeNB }\end{array}$ & $\begin{array}{l}\text { MCF Multiple Access } \\
\text { scheme: } \\
\text { * contention based: } \\
\text { EDCA } \\
\text { * coordination based: } \\
\text { MCCA }\end{array}$ \\
\hline RN PHY Layer Id & $\begin{array}{l}\text { One Id for each controlled } \\
\text { sector }\end{array}$ & One Cell Id & $\begin{array}{c}\text { Mesh Address } \\
\text { extension }\end{array}$ \\
\hline
\end{tabular}




\begin{tabular}{|c|c|c|c|}
\hline & IEEE 802.16m & LTE Advanced & IEEE 802.11s \\
\hline $\begin{array}{l}\text { Relay resource selection } \\
\text { within the frame }\end{array}$ & Configured by ABS & $\begin{array}{c}\text { Configured by higher layers } \\
\text { with using MBSFN } \\
\text { subframes }\end{array}$ & $\begin{array}{c}\text { Random Channel } \\
\text { Access }\end{array}$ \\
\hline $\begin{array}{l}\text { Relay Radio Resource } \\
\text { Scheduling is done by }\end{array}$ & ARS & Not Specified & Mesh Points (MPs) \\
\hline $\begin{array}{l}\text { Relay Resource } \\
\text { partitioning }\end{array}$ & $\begin{array}{c}\text { Time-division Transmit and } \\
\text { Receive (TTR) or } \\
\text { Simultaneous Transmit and } \\
\text { Receive (STR) }\end{array}$ & Time multiplexing & $\begin{array}{l}\text { Multiple Access } \\
\text { Multiple Channels }\end{array}$ \\
\hline HARQ & Hop by hop & Hop by hop & Not supported \\
\hline Handover & $\begin{array}{l}\text { ABS-ARS: supported } \\
\text { ARS-ARS: supported }\end{array}$ & $\begin{array}{c}\text { eNB-RN: supported } \\
\text { RN-RN: not supported }\end{array}$ & $\begin{array}{l}\text { Intra-MPP } \\
\text { Inter-MPP }\end{array}$ \\
\hline Signalling procedures & $\begin{array}{l}\text { - Topology discovery } \\
\text { - Relay path management } \\
\text { - Multicarrier operation } \\
\text { - Self organization }\end{array}$ & $\begin{array}{l}\text { - RN attach } \\
\text { - } \text { E-RAB activation / } \\
\text { modification } \\
\text { - } \text { RN startup } \\
\text { - Neighbouring } \\
\text { Information } \\
\text { - } \text { Self organization }\end{array}$ & $\begin{array}{l}\text { - Path selection and } \\
\text { forwarding } \\
\text { - Coordination } \\
\text { - Discovery } \\
\text { - Intra mesh } \\
\text { congestion control }\end{array}$ \\
\hline Security & $\begin{array}{l}\text { - Authentication: EAP } \\
\text { - Key management } \\
\text { protocol: PKM } \\
\text { - Cryptography method: } \\
\text { AES } \\
\text { - AMS privacy }\end{array}$ & $\begin{array}{l}\text { Backhaul link security } \\
\text { Access link security }\end{array}$ & $\begin{array}{l}\text { 802.11i link security: } \\
\text { - } \quad \text { MP mutual } \\
\text { authentication } \\
\text { - } \quad \text { Key management } \\
\text { - } \quad \text { Data confidentiality }\end{array}$ \\
\hline Synchronization & $\begin{array}{l}\text { midambles transmission in } \\
\text { the DL relay zone }\end{array}$ & $\begin{array}{l}\text { Control information } \\
\text { transmission inside the } \\
\text { MBSFN subframe } \\
\end{array}$ & $\begin{array}{l}\text { Beaconing and } \\
\text { synchronisation }\end{array}$ \\
\hline QoS & $\begin{array}{l}\text { - Support of a specific } \\
\text { scheduling service to } \\
\text { support real time non- } \\
\text { periodic applications } \\
\text { - Adaptive granting and } \\
\text { pooling }\end{array}$ & $\begin{array}{c}\mathrm{S} 1-\mathrm{U} \text { and } \mathrm{X} 2-\mathrm{U} \text { packets } \\
\text { mapping based on the QCI } \\
\text { associated with the UE EPS } \\
\text { bearer }\end{array}$ & Layer 2 QoS support \\
\hline Power management & Supported & Supported & Supported \\
\hline
\end{tabular}

TABLE V. SyStems MAIN FEATURES 